\title{
Linking Rings Structures and tetravalent semisymmetric graphs
}

\author{
Primož Potočnik \\ Faculty of Mathematics and Physics, University of Ljubljana \\ Jadranska 19, Ljubljana, Slovenia \\ IAM, University of Primorska, Muzejski trg 2, 6000 Koper, Slovenia \\ Stephen E. Wilson \\ Department of Mathematics and Statistics, Northern Arizona University \\ Box 5717, Flagstaff, AZ 86011, USA
}

Received 27 February 2012, accepted 27 October 2013, published online 7 August 2013

\begin{abstract}
In this paper, we introduce LR structures, a new and interesting form of symmetry in graphs. LR structures are motivated by the search for semisymmetric graphs of degree 4. We show that all semisymmetric graphs of girth and degree 4 can be constructed in a simple way from LR structures. We then show several ways in which LR structures can be constructed or found.
\end{abstract}

Keywords: Graph, automorphism group, symmetry, locally arc-transitive graph, semisymmetric graph, cycle structure, linking rings structure.

Math. Subj. Class.: 20B25, 05E18

\section{Introduction}

A regular graph $\Gamma$ is semisymmetric provided that its symmetry group is transitive on edges but not on vertices. The search for semisymmetric graphs has an intense 40-year history, beginning with Harary and Dauber's paper [9]. This unpublished work was seen by Folkman, and motivated his paper [5], which gives the smallest such graph, on 20 vertices. The earliest known semisymmetric graph, due to Marion Gray in the 1930's, shown in early dittoed versions of Foster's Census, was rediscovered by Bouwer, described in [1] and generalized in [2]. Progress on aspects of semisymmetric graphs was made in papers published

E-mail addresses: primoz.potocnik@fmf.uni-lj.si (Primož Potočnik), stephen.wilson@nau.edu (Stephen E. Wilson) 
since 1980 (see for example [4, 8, 10, 11, 12, 17, 18]). Recently, considerable attention was given to semisymmetric graphs of valence 3 [13, 14, 15], and a list of all such graphs with no more than 768 vertices was compiled in [3].

This paper continues work from [20], where the present authors began an investigation of tetravalent edge-transitive graphs, by considering those of girth at most 4 . Such a graph is either worthy or unworthy, depending on whether the neighborhoods of all pairs of distinct vertices are distinct or not. Lemma 4.3 of [20] disposes of the unworthy case by showing that every unworthy tetravalent semisymmetric graph has order $4 n$ for some integer $n$, and arises as the "subdivided double" of a dart-transitive tetravalent graph of order $n$. Note that the smallest semisymmetric graph, the Folkman graph on 20 vertices shown in Figure 1, is unworthy, and it can be obtained as a subdivided double of the complete graph $K_{5}$. Similarly, it was shown in [17, Theorem 5.2] that every semisymmetric graph on $4 p$ vertices, for $p \geq 7$ a prime, is unworthy.

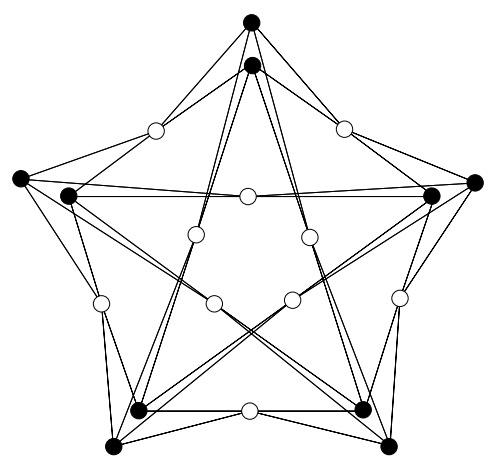

Figure 1: The Folkman graph

On the other hand, [20, Theorem 5.1(5, b)] shows that every worthy tetravalent semisymmetric graph of girth 4 and order $2 n$ is isomorphic to a graph obtained, via the "partial line graph construction", from a certain kind of cycle decomposition of a vertex-transitive tetravalent graph of order $n$. A precise statement of this result can be found in Section 5 .

The purpose of the present paper is to study worthy tetravalent semisymmetric graphs of girth 4, and the cycle decompositions from which they arise, in more detail.

\section{Preliminaries}

A standard notation for graphs is used throughout the paper: if $\Gamma$ is a graph, then $V(\Gamma)$ is its vertex set. An edge in $\Gamma$ is an unordered pair from $V(\Gamma)$ (so $\Gamma$ is a simple graph), and the set of edges is $E(\Gamma)$. An ordered pair of adjacent vertices will be called a dart (or also, an $a r c$ ). In this paper, all graphs are finite and connected. Bipartition sets in bipartite graphs are often referred to as colors, black and white, of the vertices.

A symmetry or automorphism of a graph $\Gamma$ is a permutation of its vertices which preserves adjacency. The symmetries of $\Gamma$ form a group, $\operatorname{Aut}(\Gamma)$, under composition. A graph is said to be vertex-, edge-, or dart-transitive if its automorphism group acts transitively on its vertices, edges, or darts, respectively. Dart-transitive graphs are also called symmetric or arc-transitive. 
A graph $\Gamma$ is semisymmetric provided that it is connected, regular, and $\operatorname{Aut}(\Gamma)$ is transitive on edges but not on vertices. It follows easily (see [5] for example) that such a graph $\Gamma$ is bipartite, and that $\operatorname{Aut}(\Gamma)$ is transitive on each of the two color classes of vertices.

If for every vertex $v$ of a regular graph $\Gamma$, the stabilizer $\operatorname{Aut}(\Gamma)_{v}$ acts transitively on the neighbors of $v$, then $\Gamma$ is said to be locally dart-transitive. It is well known that a locally dart-transitive graph is edge-transitive (recall that all the graphs are assumed to be connected), and that it is either dart-transitive or semisymmetric (depending on whether $\operatorname{Aut}(\Gamma)$ is transitive on the vertices or not).

\section{Cycle decompositions}

Let $\Lambda$ be a (connected) regular tetravalent graph and $\mathcal{C}$ a partition of $E(\Lambda)$ into cycles (that is, sets of edges which induce in $\Lambda$ connected subgraphs of valence 2 ). We shall call such a pair $(\Lambda, \mathcal{C})$ a cycle decomposition. For the rest of this section, let $(\Lambda, \mathcal{C})$ denote a cycle decomposition.

Two edges of $\Lambda$ will be called opposite at vertex $v$, if they are both incident with $v$ and belong to the same element of $\mathcal{C}$. The partial line graph of a cycle decomposition $(\Lambda, \mathcal{C})$ is the graph $\mathbb{P}(\Lambda, \mathcal{C})$ whose vertices are edges of $\Lambda$, and two edges of $\Lambda$ are adjacent in $\mathbb{P}(\Lambda, \mathcal{C})$ whenever they share a vertex in $\Lambda$ and are not opposite at that vertex.

The left hand side of Figure 2 shows a tetravalent graph $\Lambda$ and a cycle decomposition $\mathcal{C}$ with cycles indicated by $a-a, b-b$, etc; and the right hand side of Figure 2 shows the partial line graph of $(\Lambda, \mathcal{C})$.
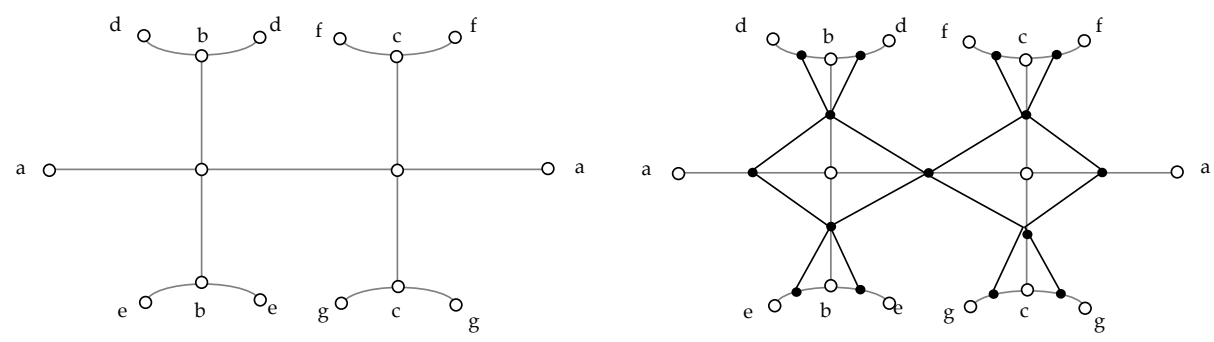

Figure 2: A cycle decomposition and its partial line graph

An isomorphism between two cycle decompositions $\left(\Lambda_{1}, \mathcal{C}_{1}\right)$ and $\left(\Lambda_{2}, \mathcal{C}_{2}\right)$ is an isomorphism $f$ of $\Lambda_{1}$ onto $\Lambda_{2}$ for which $f\left(\mathcal{C}_{1}\right)=\mathcal{C}_{2}$. An automorphism is an isomorphism from a cycle decomposition to itself. The group of automorphisms of $(\Lambda, \mathcal{C})$ will be denoted by $\operatorname{Aut}(\Lambda, \mathcal{C})$.

A cycle decomposition $(\Lambda, \mathcal{C})$ is said to be flexible provided that for every vertex $v$ and each edge $e$ containing $v$, there is a symmetry in $\operatorname{Aut}(\Lambda, \mathcal{C})$ which fixes pointwise the cycle $D \in \mathcal{C}$ containing $e$ and interchanges the other two neighbors of $v$. The edges joining $v$ to those neighbors are in some other cycle $C$ of $\mathcal{C}$, and the symmetry is called a $C$-swapper at $v$.

A cycle decomposition $(\Lambda, \mathcal{C})$ is called bipartite if $\mathcal{C}$ can be partitioned into two subsets $\mathcal{G}$ and $\mathcal{R}$ so that each vertex of $\Lambda$ meets one cycle from $\mathcal{G}$ and one from $\mathcal{R}$. Especially in constructions, we will refer to the edges of the cycles in $\mathcal{G}$ and those in $\mathcal{R}$ as green and red, 
respectively. The largest subgroup of $\operatorname{Aut}(\Lambda, \mathcal{C})$ preserving each of the sets $\mathcal{G}$ and $\mathcal{R}$ will be denoted by $\operatorname{Aut}^{+}(\Lambda, \mathcal{C})$, and we will think of it as the color-preserving group of $(\Lambda, \mathcal{C})$.

Note that in a bipartite cycle decomposition, an element of $\operatorname{Aut}(\Lambda, \mathcal{C})$ either preserves each of the sets $\mathcal{G}$ and $\mathcal{R}$ setwise, and is thus contained in $\operatorname{Aut}^{+}(\Lambda, \mathcal{C})$, or interchanges the sets $\mathcal{G}$ and $\mathcal{R}$. (In particular, in a bipartite flexible structure, swappers belong to $\operatorname{Aut}^{+}(\Lambda, \mathcal{C})$.) This shows that the index of $\operatorname{Aut}^{+}(\Lambda, \mathcal{C})$ in $\operatorname{Aut}(\Lambda, \mathcal{C})$ is at most 2. If this index is 2 , then we say that $(\Lambda, \mathcal{C})$ is self-dual. Thus $(\Lambda, \mathcal{C})$ is self-dual if and only if there is $\sigma \in \operatorname{Aut}(\Lambda, \mathcal{C})$ such that $\mathcal{G} \sigma=\mathcal{R}$ and $\mathcal{R} \sigma=\mathcal{G}$.

We can now define the central notion of this paper:

\section{LR Structures}

Definition 4.1. A cycle decomposition $(\Lambda, \mathcal{C})$ is called a linking ring structure (or briefly, an $L R$ structure) provided that it is bipartite, flexible and that $\operatorname{Aut}^{+}(\Lambda, \mathcal{C})$ acts transtively on $V(\Lambda)$.

If $(\Lambda, \mathcal{C})$ is an LR structure, then it follows from the definition that $G=\operatorname{Aut}^{+}(\Lambda, \mathcal{C})$ acts transitively on red darts, as well as on green darts, in such a way that the permutation group $G_{v}^{\Lambda(v)}$, induced by the vertex-stabiliser $G_{v}$ on the neighbourhood $\Lambda(v)$, is intransitive and is in fact permutation isomorphic to the permutation group $\langle(1,2),(3,4)\rangle \leq S_{4}$ (we shall call the permutation group $\langle(1,2),(3,4)\rangle$ the intransitive Klein 4-group). Conversely, if a connected tetravalent graph $\Lambda$ admits a vertex- but not edge-transitive group of automorphisms $G$ such that $G_{v}^{\Lambda(v)}$ is permutation isomorphic to the intransitive Klein 4-group, then the two edge-orbits of $G$ form the sets $\mathcal{G}$ and $\mathcal{R}$ of an LR structure $(\Lambda, \mathcal{C})$ for which $\mathrm{Aut}^{+}(\Lambda, \mathcal{C})$ contains $G$. This shows that, in the group-theoretical language, an LR structure could be equivalently defined as a transitive permutation group $G$ admitting two self-paired orbitals $A_{1}$ and $A_{2}$ of degree 2 such that $G_{v}^{\Lambda(v)}$ is permutation isomorphic to the intransitive Klein 4-group.

Since $\operatorname{Aut}^{+}(\Lambda, \mathcal{C})$ acts transitively on $\mathcal{G}$ and on $\mathcal{R}$, it follows that all cycles in $\mathcal{G}$ must have the same length, say $p$, and all cycles in $\mathcal{R}$ must be of the same length $q$. We then say that the LR structure $(\Lambda, \mathcal{C})$ is of type $\{p, q\}$. For a self-dual structure, of course, $p=q$.
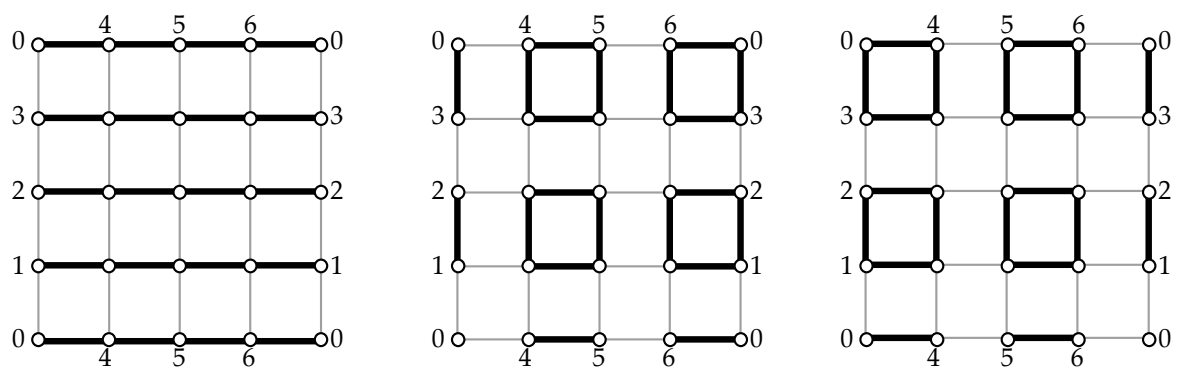

Figure 3: Three LR structures on the 4-dimensional cube $Q_{4}$, presented as a toroidal map $\{4,4\}_{4,0}$. The labels indicate the identifications of vertices and edges.

Note that a tetravalent graph can admit more than one LR structure. For example, the 4dimensional cube $Q_{4}$ (see Figure 3 ) has three distinct bi-colorings of edges, each of which 
makes it into a self-dual LR structure of type $\{4,4\}$. The three structures are isomorphic.

This leads us to pose the following open question:

Question 1: Does there exist a graph admitting non-isomorphic LR structures?

\section{Suitable LR Structures}

Let us now describe the relationship between LR structures and tetravalent edge-transitive graphs. If $(\Lambda, \mathcal{C})$ is a cycle decomposition, then a cycle $C$ in $\Lambda$ is said to be alternating if no two consecutive edges of $C$ belong to the same element of $\mathcal{C}$. In [20], a cycle decomposition $(\Lambda, \mathcal{C})$ was called smooth, provided that $\Lambda$ contained no alternating 4 -cycles, and no 3 cycles except possibly those contained in $\mathcal{C}$. Note that the latter condition on 3 -cycles is automatically satisfied for an LR structure: if $(\Lambda, \mathcal{C})$ is an LR structure, and if $b c d$ is a triangle in $\Lambda$, but not in $\mathcal{C}$, then two of the three edges are the same color; say $b c$ and $c d$ are red, while $b d$ is green. Then a swapper at $b$ which fixes the red cycle containing $b c d$ would interchange the two green neighbors at $b$. But one of these is $d$, which is left fixed by this symmetry. This is a contradiction, and so no such triangle can exist.

Definition 5.1. An LR structure which is not self-dual and contains no alternating 4-cycles is called suitable.

It is not difficult to see that a cycle decomposition $(\Lambda, \mathcal{C})$ is a suitable LR structure if and only if it satisfies the conditions stated in [20, Theorem 5.1(5, b)]. This theorem, combined with Lemma 6.3, which will be proved in the following section, implies the following correspondence, which explains the relevance of LR structures to the study of tetravalent edge-transitive graphs.

Theorem 5.2. The partial line graph construction $\mathbb{P}$ induces a bijective correspondence between the set of LR structures and the set of worthy bipartite locally dart-transitive tetravalent graphs of girth 4 . If $(\Lambda, \mathcal{C})$ is an LR structure, then $\mathbb{P}(\Lambda, \mathcal{C})$ is semisymmetric if and only if $(\Lambda, \mathcal{C})$ is suitable; and is arc-transitive otherwise. If $(\Lambda, \mathcal{C})$ contains an alternating 4 -cycles, then $\mathbb{P}(\Lambda, \mathcal{C})$ is a skeleton of an arc-transitive map of type $\{4,4\}$ on the torus.

Remark. If a graph $\Lambda$ has a cycle decomposition $\mathcal{C}$ for which $(\Lambda, \mathcal{C})$ is a suitable $\mathrm{LR}$ structure, it is conceivable that it might allow a different cycle decomposition $\mathcal{C}^{\prime}$ for which $\left(\Lambda, \mathcal{C}^{\prime}\right)$ is an LR structure. We know of no such example, and we conjecture that none exist. Proving that the conjecture holds would allow us to simplify our notation, and talk about an $L R$ graph $\Lambda$. We will discuss this conjecture further in our final section.

Every new LR structure gives a new edge-transitive graph, and so we are interested in finding and creating LR structures. The aim of this paper is to provide constructions of LR structures (and thus of bipartite tetravalent edge-transitive graphs), which show how varied the LR structures can be.

\section{Basic constructions}

We begin our investigation of LR structures by presenting some basic contructions.

\subsection{LR Structures on the wreath graphs}

The wreath graph $W(n, 2)$ is defined to be the graph on $2 n$ vertices $u_{i}, v_{i}$, for $i \in \mathbb{Z}_{n}$, with all edges from vertices of subscript $i$ to those of subscript $i+1$. Alternatively, $W(n, 2)$ 
can be defined as the lexicographic product of the cycle $C_{n}$ with the edgeless graph on two vertices.

Construction 6.1. If $n$ is even, let $\Lambda$ be the wreath graph $W(n, 2)$ and let $\mathcal{C}$ be the set of 4 -cycles of $W(n, 2)$ of the form $\left[u_{i}, u_{i+1}, v_{i}, v_{i+1}\right]$. Color such a 4 -cycle red if $i$ is even and green if $i$ is odd. Then $(\Lambda, \mathcal{C})$ is clearly a self-dual LR-structure of type $\{4,4\}$.

The partial line graphs of the LR-structures on the wreath graphs defined above are thus arc-transitive tetravalent graphs, which may also be obtained as 2-fold covers over the wreath graphs. These graphs have appeared in the literature before, for example in [6, 7].

\subsection{Toroidal LR Structures}

Consider the LR structure on the cube $Q_{4}$ depicted in the left-hand side of Figure 3. This structure can be generalized to other maps on the torus:

Definition 6.2. Let $\mathcal{M}$ be a map of type $\{4,4\}$ on the torus. Then $\mathcal{M}$ arises as a quotient $\{4,4\}_{T}$ of the tessellation $\{4,4\}$ by a group $T$ of translations. Color edges of the tessellation belonging to one parallel class green, the other edges red. Since translations preserve these colors, we can consider the edges of $\mathcal{M}$ colored in the same way. If the group $T$ is normalized by horizontal and vertical reflections, we will say that $\mathrm{T}$ is reflective. In this case, then those reflections act on $\mathcal{M}$ as well, giving the structure its swappers. In any map of type $\{4,4\}$ on the torus, the group of translations is transitive on vertices, and so if $T$ is reflective, the resulting graph $\{4,4\}_{T}$ is an LR structure, and we will call it a toroidal LR structure.

Note that if $(\Lambda, \mathcal{C})$ is a toroidal LR structure, then $\mathbb{P}(\Lambda, \mathcal{C})$ is a bipartite edge-transitive skeleton of a map of type $\{4,4\}$ on the torus.

Remark: The possibilities for the group $T$ of translations satisfying the requirement that $T$ is normalized by all horizontal and vertical reflections are exactly these two:

(I) $T$ is generated by $(b, 0)$ and $(0, c)$ for some $b$ and $c$ both at least 3 . The resulting graph has $b c$ vertices and is of type $\{b, c\}$. We will call this structure $\{4,4\}_{[b, c]}$.

(II) $T$ is generated by $(b, c)$ and $(b,-c)$ for some $b$ and $c$ both at least 2 . The resulting graph has $2 b c$ vertices and is of type $\{2 b, 2 c\}$. We will call this structure $\{4,4\}_{<b, c>}$.

These two types are shown in Figure 4; on the left is $\{4,4\}_{[4,3]}$, and on the right is $\{4,4\}<3,2>$.

Observe that every toroidal LR structure, as defined above, has alternating 4-cycles. Surprisingly, the converse holds as well.

Lemma 6.3. An LR structure which has an alternating 4-cycle is toroidal and arises as in Definition 6.2.

Proof. Suppose that $a, b, c, d$ is an alternating 4-cycle in which $\{a, b\}$ and $\{c, d\}$ are the green edges, $\{b, c\}$ and $\{d, a\}$ the red. Then a green swapper at $a$ fixes $a$ and $d$ but not $b$, and so sends $a b c d$ to a different alternating 4-cycle containing the red edge $\{d, a\}$. Thus every red edge belongs to at least two alternating 4-cycles, and similarly, so does every green. Now if any edge belongs to more than two alternating 4-cycles, it is not hard to show that the structure must be the toroidal structure for the map $\{4,4\}_{2,2}$ (its underlying 

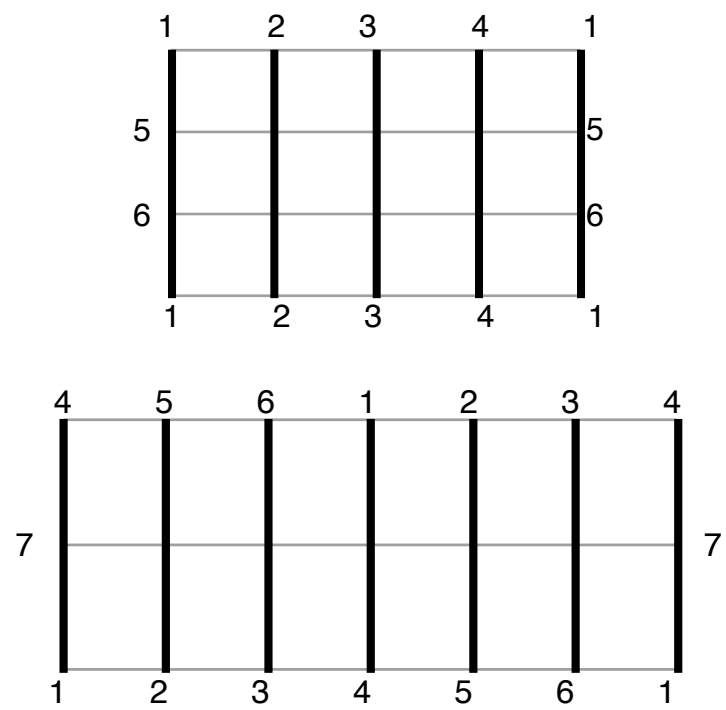

Figure 4: Two kinds of toroidal LR structures

graph is $K_{4,4}$ ). If not, then every edge belongs to exactly two alternating 4-cycles. Using these cycles as faces constructs a map on a surface. The map has quadrangular faces, and four meet at every vertex and so the Euler characteristic of the surface is 0 . The surface is thus the torus or the Klein bottle. The results of the paper [21] show that no map on the Klein bottle can have swappers except for some whose skeleton is not a simple graph, and therefore the map and structure must be toroidal.

This lemma, combined with [20, Theorem 5.1(5, b)], proves Theorem 5.2

\subsection{Barrels}

In this section, we introduce our first families of suitable LR structures. The barrels $\operatorname{Br}(k, n ; r)$ and the mutant barrels $\operatorname{MBr}(k, n ; r)$ are, of all known LR structures, the simplest to describe, the easiest to visualize and the most common to occur.

The structure $\operatorname{Br}(k, n ; r)$ is defined for $k$ even, $k \geq 4, n \geq 5$, and $r^{2}= \pm 1 \bmod n$ but not $r= \pm 1 \bmod n$. It has vertices $\mathbb{Z}_{k} \times \mathbb{Z}_{n}$, with $(i, j)$ red-adjacent to $(i \pm 1, j)$ and green-adjacent to $\left(i, j \pm r^{i}\right)$. We may and usually do assume that $0 \leq r<\frac{n}{2}$.

We should mention that the underlying graphs of the LR structures $\mathrm{Br}(k, n ; r)$ have appeared in different contexts under different names before, most recently in [16], where the class of $\mathcal{Y}$ graphs include the graph $\operatorname{Br}(k, n ; r)$ as $\mathcal{Y}(k, n, r, 0)$.

Figure 5 shows part of such a graph having $n=5$ and $r=2$, with red edges shown thin, green edges shown bold.

$\operatorname{Br}(k, n ; r)$, has color-preserving symmetries $(i, j) \mapsto(i, j+1)$ and $(i, j) \mapsto(i+1, r j)$, which show it to be vertex transitive. In the stabilizer of $(0,0)$ are symmetries $(i, j) \mapsto$ $(-i, j)$ and $(i, j) \mapsto(i,-j)$ which act as swappers there. Thus, $\operatorname{Br}(k, n ; r)$ is an LR 


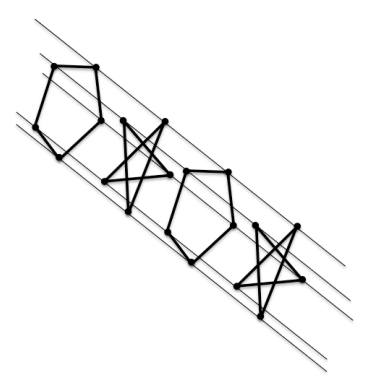

Figure 5: part of $\operatorname{Br}(k, 5 ; 2)$

structure.

A related graph is $\operatorname{MBr}(k, n ; r)$, which is defined if both $k$ and $n$ are even, $k \geq 2$, $n \geq 6$, and $r^{2}= \pm 1 \bmod n$ but not $r= \pm 1 \bmod n$. It has vertices $\mathbb{Z}_{k} \times \mathbb{Z}_{n}$, with $(i, j)$ red-adjacent to $(i+1, j)$ for $0 \leq i<k-1,(k-1, j)$ red-adjacent to $(0, j+n / 2)$ and $(i, j)$ green-adjacent to $\left(i, j \pm r^{i}\right)$ for all $i$.

$\operatorname{MBr}(k, n ; r)$ has symmetries that are swappers with definitions similar to those for $\operatorname{Br}(k, n ; r)$. Thus, every member of these two families is an LR structure.

For most parameters $k, n$ and $r$, the LR structures $\operatorname{Br}(k, n ; r)$ and $\operatorname{MBr}(k, n ; r)$ are suitable. Each one has $k n$ vertices and is of type $\{k, n\},\{2 k, n\}$, respectively.

We are now ready to determine which barrels give rise to suitable LR-structures.

Theorem 6.4. Each of the LR structures $\operatorname{Br}(k, n ; r), M B r(k, n ; r)$ has $k n$ vertices and is of type $\{k, n\},\{2 k, n\}$, respectively.

The LR structures $\operatorname{Br}(k, n ; r)$ are suitable for all admissible parameters: $k \geq 4$ even, $n \geq 5$, and $2 \leq r<\frac{n}{2}, r^{2} \equiv \pm 1(\bmod n)$.

The LR structure $\operatorname{MBr}(k, n ; r)$ is suitable for all admissible parameters: $k \geq 4$ even, $n \geq 6$ even, $2 \leq r<\frac{n}{2}, r^{2} \equiv \pm 1(\bmod n)$, with the exceptions of $M B r(k, 2 k ; k-1)$ for $k$ even, $k \geq 4$, which is self-dual; and $\operatorname{MBr}\left(2, n ; \frac{n}{2} \pm 1\right)$, which has alternating 4-cycles.

Proof. The claim about the type of $\operatorname{Br}(k, n ; r)$ and $M B r(k, n ; r)$ follows immediately from the definition of the two structures. It is also clear that no member of either family has an alternating 4-cycle, except in the case of $\operatorname{MBr}\left(2, n ; \frac{n}{2} \pm 1\right)$; here, an alternating 4-cycle is $(0,0) \sim(0,1) \sim\left(1,1+\frac{n}{2}\right) \sim\left(1,1+\frac{n}{2}+r\right)=(1,0) \sim(0,0)$.

Number the red cycles $1,2, \ldots$ in the order in which they appear around a fixed green cycle, and do the same for green cycles. In $\operatorname{Br}(k, n ; r)$, every green cycle appears once around each red cycle and vice versa; in $\operatorname{MBr}(k, n ; r)$, every green cycle appears twice around each red cycle and vice versa. Then in every red cycle, the green cycles appear in the same order, $1,2, \ldots$, while around alternating green cycles, they appear in the orders $1,2,3 \ldots$ and $1,1+r, 1+2 r, \ldots$ In most cases, these are different orders, and so no symmetry can switch red with green. The only way that these can be the same order is if:

(1) each number appears twice, so the graph is the underlying graph of a mutant barrel LR structure,

(2) the cycles have the same length, so that $n=2 k$, 
(3) $r+1 \equiv 2 \bmod k$, so that $r=k+1$

It remains to show that $\operatorname{MBr}(k, 2 k ; k-1)$ is indeed self-dual. To do that, we observe that it is isomorphic to the following LR structure: vertices are elements of $\mathbb{Z}_{k} \times \mathbb{Z}_{k} \times \mathbb{Z}_{2}$; red edges are of the form:

- $(i, j, d) \sim(i+1, j, d), \quad 0 \leq i<k-1$

- $(k-1, j, d) \sim(0, j, d+1)$,

while green edges are of the form:

- $(i, j, d) \sim(i, j+1, d+i), \quad 0 \leq j<k-1$

- $(i, k-1, d) \sim(i, 0, d+1+i)$,

The function $\varphi:(i, j, r) \mapsto(i, j+r k)$ is an isomorphism from this structure to the structure $\operatorname{MBr}(k, 2 k ; k-1)$. In this form, it is easy to check that $(i, j, r) \mapsto(j, i, r+i j)$ is a color-reversing symmetry of the structure. Thus, $\operatorname{MBr}(k, 2 k ; k-1)$ is self-dual and so not suitable.

We conclude this section by mentioning that the smallest suitable barrel $\operatorname{Br}(4,5 ; 2)$ gives rise to the the semisymmetric graph $\mathbb{P}(B r(4,5 ; 2))$, which has 40 vertices, 80 edges, and the automorphism group of which has order 80 . This appears to be the smallest automorphism group of any semisymmetric graph. There is a second semisymmetric graph of the same size whose group also has size 80 . This graph is $\mathbb{P}(\operatorname{MBr}(2,10 ; 3))$.

\section{Quotients and covers}

Quotients and covers of graphs have become a standard tool in studying vertex-transitive graphs, and can be used almost without any change in the setting of LR structures as well. Here we only briefly summarize the basic ideas of the covering techniques.

Let $\Lambda$ be a graph, and let $\mathcal{B}$ be a partition of the vertex-set $V(\Lambda)$. Then the quotient graph $\Lambda_{\mathcal{B}}$ is the graph with $\mathcal{B}$ as its vertex-set and with two elements $B_{1}, B_{2} \in \mathcal{B}$ adjacent in $\Lambda_{\mathcal{B}}$ whenever there is an edge in $\Lambda$ between some $v_{1} \in B_{1}$ and some $v_{2} \in B_{2}$. If the partition $\mathcal{B}$ is invariant under some $G \leq \operatorname{Aut}(\Lambda)$, then $G$ acts in a natural way on $\Lambda_{\mathcal{B}}$ by automorphisms. If there are no edges within the elements of $\mathcal{B}$ and if the graph induced by two adjacent elements of $\mathcal{B}$ is a perfect matching, then $\Lambda$ is called a cover of $\Lambda_{\mathcal{B}}$.

An important special case of quotients and covers arises in the following setting. Let $G$ be a vertex-transitive group of automorphisms of a connected graph $\Lambda$, and let $N$ be a normal subgroup of $G$ acting semiregularly on $V(\Lambda)$ (i.e. $N_{v}=1$ for every $v \in V(\Lambda)$ ). Then the orbits of $N$ form a $G$-invariant partition of $V(\Lambda)$. The corresponding quotient graph is then called a $G$-normal quotient of $\Lambda$ by $N$ and denoted by $\Lambda_{N}$. If in addition $\Lambda$ is a cover of $\Lambda_{N}$, then it is called a regular cover. In this case, the action of $G$ on $\Lambda_{N}$ induces a faithful vertex-transitive action of $G / N$ on $\Lambda_{N}$, and we say that $G \leq \operatorname{Aut}(\Lambda)$ is a lift of $G / N \leq \operatorname{Aut}\left(\Lambda_{N}\right)$

All these notions extend naturally to LR structures. In particular, if $G \leq \operatorname{Aut}(\Lambda, \mathcal{C})$ and $\mathcal{B}$ is a $G$-invariant partition of $V(\Lambda)$ such that $\Lambda$ is a cover of $\Lambda_{\mathcal{B}}$, then one can define an LR structure $\left(\Lambda_{\mathcal{B}}, \mathcal{C}_{\mathcal{B}}\right)$ in an obvious way. For example, take any of the three LR structures on $Q_{4}$ depicted in Figure 3, and let $\mathcal{B}$ be a partition of $V\left(Q_{4}\right)$ into pairs of antipodal 
vertices in $Q_{4}$. The corresponding quotient graph $\left(Q_{4}\right)_{\mathcal{B}}$ is isomorphic to $K_{4,4}$, and $Q_{4}$ is a cover of the quotient. In fact, letting $N$ be the group generated by the automorphism of $Q_{4}$ interchanging the pairs of antipodal vertices, we see that $\left(Q_{4}\right)_{\mathcal{B}}=\left(Q_{4}\right)_{N}$ is a normal quotient of $Q_{4}$. Clearly, $\mathcal{B}$ is invariant under $\operatorname{Aut}\left(Q_{4}\right)$, and so the three (isomorphic) LR structures on $Q_{4}$ induce three isomorphic LR structures on $K_{4,4}$.

\subsection{Loose and antipodal attachments}

Consider two cycles, $C_{1}$ and $C_{2}$, of an LR structure $(\Lambda, \mathcal{C})$, one green and one red, which intersect in a common vertex $v$. If $v$ is the only vertex in their intersection, then the vertextransitivity of the automorphism group implies that any two intersecting cycles meet in exactly one vertex. Following the language of [19], we will say in this case that the LR structure is loosely attached.

On the other hand, if there is another vertex $u$ in the intersectection of $C_{1}$ and $C_{2}$, then the existence of swappers at $v$ implies that $u$ is the vertex which is antipodal to $v$ on $C_{1}$ and $C_{2}$ (in particular, $C_{1}$ and $C_{2}$ are of even length). If this is the case, then by vertextransitivity, any two intersecting cycles intesect in exactly two vertices, located antipodally on the intersecting cycles. LR structures of this type will be called antipodally attached.

Of course, if $(\Lambda, \mathcal{C})$ is an antipodally attached LR structure, then the permutation swapping the two vertices in each antipodal pair is a central involution in $\operatorname{Aut}(\Lambda, \mathcal{C})$, and thus generates a normal subgroup $N \leq \operatorname{Aut}(\Lambda, \mathcal{C})$. Unless the type of $(\Lambda, \mathcal{C})$ is $\{4, q\}$ or $\{p, 4\}$, identifying pairs of antipodal vertices projects $(\Lambda, \mathcal{C})$ onto a loosely attached LR structure $\left(\Lambda_{N}, \mathcal{C}_{N}\right)$ and the projection is a regular covering projection.

We already have examples of both kinds of attachments and the corresponding projections. Each toroidal LR structure $\{4,4\}_{\langle b, c\rangle}$ is antipodally attached, and the projection sends it onto $\{4,4\}_{[b, c]}$ (if $b$ and $c$ are both at least 3). Each $\operatorname{MBr}(2 k, 2 n ; r)$ is antipodally attached, and the projection sends it onto $\operatorname{Br}(k, n ; r)$ (if $k$ and $n$ are both at least 3).

If $(\Lambda, \mathcal{C})$ is an antipodally attached LR structure of type $\{4,4\}$, then it is easy to see that $\Lambda$ is a wreath graph with $\mathcal{C}$ the decomposition presented in Construction 6.1.

Antipodally attached LR structures of type $\{4, q\}$ for some $q>4$ will be studied in a future paper, where we show that such a structure arises from a certain cycle decomposition, called a "cycle structure", in a smaller tetravalent dart-transitive graph, thus allowing a reduction to smaller graphs.

The above discussion is summarized in the following theorem.

Theorem 7.1. Let $(\Lambda, \mathcal{C})$ be an antipodally attached LR structure of type $\{p, q\}$. If $p=$ $q=4$, then $(\Lambda, \mathcal{C})$ is the type $\{4,4\}$ LR structure on a wreath graph $W(n, 2)$ described in Construction 6.1. If $p, q \geq 6$, then $(\Lambda, \mathcal{C})$ arises as a 2 -fold cover of a loosely attached LR-structure.

Question 2: For which loosely attached LR structures does an antipodally attached cover exist?

Question 3: How is such a cover constructed?

Question 4: If $(\Lambda, \mathcal{C})$ is suitable and antipodally attached, when is the factor structure suitable? 


\section{The Big question}

In Section 5, we conjectured that a suitable LR structure is unique on its graph. We believe that a stronger conjecture holds:

Conjecture 8.1. If $(\Lambda, \mathcal{C})$ is an $\operatorname{LR}$ structure for which $\operatorname{Aut}^{+}(\Lambda, \mathcal{C})$ is a proper subgroup of $\operatorname{Aut}(\Lambda)$, then it is self-dual.

Certainly, if $\mathrm{Aut}^{+}(\Lambda, \mathcal{C})$ is a proper subgroup of $\operatorname{Aut}(\Lambda, \mathcal{C})$, then $(\Lambda, \mathcal{C})$ is self-dual. So we may suppose that $\operatorname{Aut}(\Lambda)$ contains some $\sigma$ which sends some red edges to red edges and some red edges to green edges. The conjecture would imply that $(\Lambda, \mathcal{C})$ is self-dual. While that conjecture is still open, we can and will prove that such an LR structure must be of a self-dual type.

Theorem 8.2. If $(\Lambda, \mathcal{C})$ is an LR structure of type $\{p, q\}$ and $\operatorname{Aut}(\Lambda)$ contains some $\sigma$ which does not preserve $\mathcal{C}$, then $p=q$.

Proof. We will actually prove that under the hypothesis, some symmetry must send some red cycle to a green cycle. Suppose not. Let $m$ be maximal such that some $\sigma$ in $\operatorname{Aut}(\Lambda)$ sends some $m$ consecutive edges of a red cycle to green edges. Then $m$ must be less than both $p$ and $q$. Suppose that $u_{0}, u_{1}, \ldots, u_{m-1}, u_{m}, u_{m+1}, \ldots$ are consecutive vertices on some red cycle, $v_{0}, v_{1}, \ldots, v_{m-1}, v_{m}, v_{m+1}, \ldots$ are consecutive vertices on some green cycle, and that for $i=0,1,2 \ldots, m, u_{i} \sigma=v_{i}$. Suppose that $a, b$ are the green neighbors of $u_{m}$, that $c, d$ are the red neighbors of $v_{m}$. Finally, let $\mu$ be a green swapper at $u_{m}$ and $\tau$ be a red swapper at $v_{m}$, as shown in Figure 6.

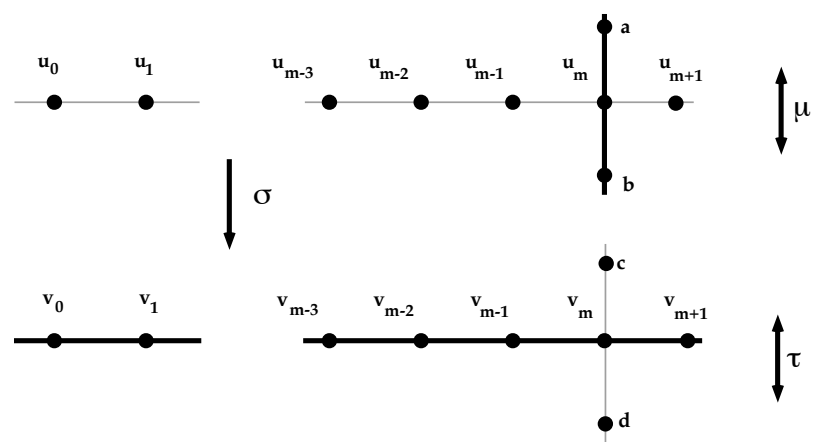

Figure 6: A color-mixing symmetry $\sigma$

Because $m$ is maximal, we know that $u_{m+1} \sigma \neq v_{m+1}$. Assume, without loss of generality, that $u_{m+1} \sigma=c, a \sigma=v_{m+1}, b \sigma=d$. Consider $\sigma^{\prime}=\sigma \tau \sigma^{-1} \mu \sigma$. Then for $i=0,1,2, \ldots, m, \mu$ fixes $u_{i}$ and $\tau$ fixes $v_{i}$ and so $u_{i} \sigma^{\prime}=v_{i}$. And $u_{m+1} \sigma^{\prime}=$ $u_{m+1} \sigma \tau \sigma^{-1} \mu \sigma=c \tau \sigma^{-1} \mu \sigma=d \sigma^{-1} \mu \sigma=b \mu \sigma=a \sigma=v_{m+1}$, contradicting the maximality of $m$. Thus $p=q$.

The techniques of this proof will extend to prove the existence of a symmetry which sends one red cycle and all of its green neighbors to a green cycle and all of its red neighbors, but we cannot see how to push the technique any farther.

In future papers, we will show both algebraic and combinatorial constructions for LR structures. 


\section{References}

[1] I. Z. Bouwer, An edge but not vertex transitive cubic graph, Bull. Can. Math. Soc. 11 (1968), 533-535.

[2] I. Z. Bouwer, On edge but not vertex transitive regular graphs, J. Combin. Theory, Ser. B 12 (1972), 32-40.

[3] M. D. E. Conder, A. Malnič, D. Marušič, and P. Potočnik, A census of semisymmetric cubic graphs on up to 768 vertices, J. Algebraic Combin. 23 (2006), 255-294.

[4] S. F. Du, F. R. Wang, and L. Zhang, An infinite family of semisymmetric graphs constructed from affine geometries, European J. Combin. 24 (2003), 897-902.

[5] J. Folkman, Regular line-symmetric graphs, J. Combin. Theory 3 (1967), 215-232.

[6] A. Gardiner and C. Praeger, On 4-valent symmetric graphs, European J. Combin. 15 (1994), 375-381.

[7] A. Gardiner and C. Praeger, A characterization of certain families of 4-valent symmetric graphs, European J. Combin. 15 (1994), 383-397.

[8] D. Goldschmidt, Automorphisms of trivalent graphs, Ann. Math. 111 (1980), 377-406.

[9] F. Harary and E. Dauber, Line-symmetric but not point-symmetric graphs, unpublished and apparently irrecoverable.

[10] M. E. Iofinova and A. A. Ivanov, Biprimitive cubic graphs, Investigations in Algebraic Theory of Combinatorial Objects, (Proceedings of the seminar, Institute for System Studies, Moscow, 1985) Kluwer Academic Publishers, London, 1994, 459-472.

[11] A. V. Ivanov, On edge but not vertex transitive regular graphs, Ann. Discrete Math. 34 (1987), 273-286.

[12] F. Lazebnik and R. Viglione, An infinite series of regular edge- but not vertex-transitive graphs, J. Graph Theory 41 (2002), 249-258.

[13] Z. Lu and M.Y. Xu, Cubic semisymmetric graphs constructed from Cayley graphs of degree 4, Graph Theory Notes N. Y. 44 (2003), 44-51.

[14] A. Malnič, D. Marušič, and C.Q. Wang, Cubic edge-transitive graphs of order $2 p^{3}$, Discrete Math. 274 (2004), 187-198.

[15] A. Malnič, D. Marušič, P. Potočnik, and C.Q. Wang, An infinite family of cubic edge- but not vertex-transitive graphs, Discrete Math. 280 (2004), 133-148.

[16] D. Marušič and P. Šparl, On Quartic Half-arc-transitive Metacirculants, J. Alg. Combin. 28 (2008), 365-395.

[17] D. Marušič and P. Potočnik, Semisymmetry of generalized Folkman graphs, European J. Combin. 22 (2001), 333-349.

[18] D. Marušič and P. Potočnik, Bridging semisymmetric and half-arc-transitive actions on graphs, European J. Combin. 23 (2002), 719-732. J. Graph Theory, (2000).

[19] D. Marušič and C. Praeger, Tetravalent Graphs Admitting Half-Transitive Group Actions: Alternating Cycles, J. Comb. Theory, Ser. B 75 (1995), 188-205.

[20] P. Potočnik and S. Wilson, Tetravalent edge-transitive graphs of girth at most 4, J. Comb. Theory, Ser. B 97 (2007), 217-236.

[21] S. Wilson, Uniform maps on the Klein bottle, J. for Geom. and Graphics 10 (2006), 161-171. 